\title{
Relativization in Italian Sign Language: the missing link of relativization
}

\author{
Carlo Cecchetto and Caterina Donati
}

\begin{abstract}
Relativization in Italian Sign Language (LIS) is one of those still rather few cases in the field of sign language syntax where there is some literature, a number of diverging analyses, and some debate. This literature is the base for this article. Its aim is to reconcile the opposing views proposed under a new general analysis of relativization, which the authors have been developing in recent years based on spoken languages (Cecchetto \& Donati 2010, 2015; Donati \& Cecchetto 2011). In a nutshell, relatives are seen as relabeling structures, where the movement of a nominal element nominalizes the clause. While in externally headed structures, as in English, what moves is the pivot Noun, in LIS a determiner (glossed PE) performs this relabeling movement, leaving the head Noun stranded in situ. This makes LIS relatives very similar to free relatives. Some cross-linguistic perspectives are discussed.
\end{abstract}

\section{Introduction}

Relativization in Italian Sign Language (Lingua dei Segni Italiana, LIS ${ }^{1}$ ) is one of those still rather few cases in the field of sign language linguistics where there is some literature, a number of diverging analyses, and some debate. This literature and this disagreement will constitute the base for this article. We refer in particular to two 'families' of analyses: The first, pioneering discovery of a relativization strategy in LIS by the Milan group (Carlo Cecchetto, Carlo Geraci, and Sandro Zucchi), described in Cecchetto, Geraci \& Zucchi (2006); and the alternative approach pursued by Chiara Branchini in her thesis (Branchini 2007; also see Branchini 2014), which is summarized and further developed in Branchini \& Donati (2009).

The aim of this chapter is to reconcile the opposing views proposed in these papers under a new general analysis of relativization. We will first start 
from what we know about relativization in LIS and by briefly summarizing the competing analyses (Section 2). We will then introduce a new approach to relativization, which the authors of this paper have been developing in recent years based on spoken languages (Cecchetto \& Donati 2010, 2015; Donati \& Cecchetto 2011), as it will provide the frame for the proposed analysis (Section 3). We will then go back to LIS relativization (Section 4), showing that an analysis based on this framework predicts all the properties displayed by the LIS construction. Some additional evidence will be discussed, concerning in particular a minimally different construction. A conclusion (Section 5) closes the article, tentatively putting LIS clauses in a typological perspective, where they fill a missing link which is predicted to exist by the framework adopted.

An important proviso should be introduced before starting: This paper contains very few new data, but mostly capitalizes on the data originally gathered and described in the papers cited above, and especially in Branchini (2007, 2014), thus heavily relying on them. Most of the data contained in this article correspond to video clips originally recorded by the researchers quoted here and can be viewed in the sites to which the quoted papers link. All data come from deaf native signers of LIS.

\section{What we know about relativization in LIS}

LIS displays a specialized structure for relativization, which has been described extensively by Cecchetto, Geraci \& Zucchi (2006), Branchini (2007, 2014), and Branchini \& Donati (2009). It essentially consists of two clauses: A first clause always (or mostly) closed by a sign we will gloss PE (the PE-clause), coupled with a second clause (the main clause) containing a gap or a IX interpreted anaphorically with an NP in the PE-clause (the head). In (1), to illustrate, the head is DOG. The sign PE is realized with the index finger stretching out and shaken downwards and its conventional name derives from the fact that it is typically co-articulated with bilabial phonemes as $p$. In (1) and in other glosses below, we explicitly indicate subject and object spatial agreement on the verb by using subscripts, if this facilitates the reader. Subscripts are also used for signaling spatial agreement, as we will see below.

br

(1) YESTERDAY CAT DOG $_{\mathrm{i} i} \mathrm{CHASE}_{\mathrm{k}}$ PE $_{\mathrm{i}}\left(\mathrm{IX}_{\mathrm{i}}\right)$ RETURN HOME DONE 'The dog that chased the cat returned home.' 
This structure minimally differs from a coordinate clause as (2) only for two properties: (i) the presence of PE, which never shows up in coordination; (ii) a specific non-manual marking, here glossed as 'br' (brow raise), obligatorily realized in relatives (1), but absent in coordination (coordination is signaled by different non-manual markings).

(2) CAT $_{k}$ DOG $_{i j}$ CHASE $_{k}$ HOME IX ${ }_{i}$ RETURN

'The dog chased the cat and returned home.'

We now briefly summarize some properties of PE-clauses.

\subsection{PE-clauses are internally headed}

Cecchetto, Geraci \& Zucchi (2006) have established that PE-clauses are head internal. This can be shown by looking at the distribution of time adverbs like YESTERDAY, which are always found in clause-initial position in LIS. The head noun never precedes a time adverbial in a PE-clause. In (3), YESTERDAY precedes the head HOUSE, while the reverse order would be ungrammatical.

br

(3) YESTERDAY HOUSE MARIA SEE PE $_{\mathrm{i}}$ TODAY BURN 'The house that Maria saw yesterday is on fire today.'

\subsection{The nature of PE}

Branchini $(2007,2014)$ and Branchini \& Donati (2009) have shown that PE is a demonstrative/determiner-like element whose distribution is not restricted to relatives. In (4) to (7), for example, PE acts a nominalizer in combination with adjectives and possessives.

(4) LAST PE

'the last one'

(5) POSS PE

'mine'

(6) SMALL PE

'the little one'

(7) RED PE

'the red one' 


\subsection{The position of $\mathrm{PE}$}

PE belongs to the embedded clause and marks its right edge (hence the label PE-clause). It is not part of the main clause. This can be shown capitalizing once again on the rigid position of time adverbials in LIS (Cecchetto, Geraci \& Zucchi 2006): In (8) PE precedes a time adverbial like TODAY, which marks the left edge of the main clause. The reverse order would be ungrammatical. The boundary between the PE-clause and the main clause is also identified by prosodic cues: The 'br' non-manual marking stops before the main clause starts and an intonational break occurs.

(8) YESTERDAY HOUSE MARIA SEE PE $_{\mathrm{i}}$
'The house that Maria saw yesterday is on fire today.'

Notice that the right edge of the clause corresponds to the Complementizer area in LIS (Cecchetto, Geraci \& Zucchi 2009): This is where wh-elements ultimately surface, and where other COMP related elements (such as Focus) are located. More generally, LIS belongs quite coherently to the head final typology: SOV is the preferred word order (cf. Branchini \& Geraci (2011) for discussion), and functional heads such as the aspectual marker FATTO (cf. Zucchi et al. 2010), negation, and modals follow the verb.

\subsection{The movement of PE}

There is evidence that PE is not directly merged in this position in the COMP area, but it is rather moved there from a position close to the relative clause head: In other words, PE starts as the determiner of the head and moves to the $\mathrm{C}$ area (see Branchini \& Donati (2009) for details), as schematized in (9b). This is shown by the fact that (i) PE obligatorily agrees in space with the head noun, as indicated by co-indexing in the glosses; and (ii) PE can optionally be realized in situ, at least according to some speakers, as illustrated in (9a).

br

(9) a. GIRL PE $_{\mathrm{i}}$ LEAVE BRING SUITCASE

'The girl that left brought the suitcase.'

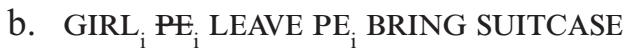

'The girl that left brought the suitcase.' 
Interestingly, PE can never pied-pipe anything in its movement to COMP: In particular, it cannot pied-pipe the head, as shown by the ungrammaticality of (10).

* YESTERDAY MARIA SEE HOUSE PE $_{\mathrm{i}}$ TODAY BURN

(intended meaning: 'The house that Maria saw yesterday is on fire today.')

2.5. The spreading of the non-manual marking

As for the non-manual marking obligatorily present in PE-clauses, either it is concentrated locally on PE itself (11); or, more frequently, it spreads and, if it does so, it typically spreads over the entire clause (12).

(11) DOG $_{\mathrm{i}} \mathrm{CAT}_{\mathrm{j}} \mathrm{CHASE}_{\mathrm{j}} \underline{\mathrm{PE}}_{\mathrm{i}}$
'The dog that chased the cat came home.'

br

(12) $\operatorname{DOG}_{i} \mathrm{CAT}_{\mathrm{j} i} \mathrm{CHASE}_{\mathrm{j}} \mathrm{PE}_{\mathrm{i}}$ HOME RETURN

'The dog that chased the cat came home.'

2.6. The interpretation is not (always) restrictive

Finally, it has been noticed that PE-clauses do not always behave as restrictive relatives in interpretation. In particular, Cecchetto, Geraci \& Zucchi (2006) observe the following: When the head is quantified, as in (13), the interpretation of the construction strongly diverges from its English counterpart.

(13) $\frac{\text { br }}{\text { ALL BOYS }_{\mathrm{i}} \text { LEAVE PE }}$ $_{\mathrm{i}} \quad$ THEY $_{\mathrm{i}}$ CALL

As discussed extensively with the informants, (13), unlike the English sentence 'All the boys that left called', entails that all the boys called (and left). This is indeed the kind of interpretation that we expect to find in appositive relative clauses in English, such as (14).

(14) All the boys, who left, called. 
This is one of the area where the two analyses diverge: Cecchetto, Geraci \& Zucchi (2006) conclude from this and other similar interpretive facts, including the observation that a negative quantifier cannot be the head of a PE-clause, that LIS PE-clauses are non-restrictive and offer a semantic account for this property. Branchini \& Donati (2009) go against this view, running a number of (mostly syntactic) tests to argue that LIS PE-clauses are indeed restrictive, and try to derive interpretative facts such as (13) from some other sources. For example, they notice that in (13), the quantifier sits inside the PE-clause and this position of the quantifier, rather than the intrinsic nature of PE-clauses, might explain the non-restrictive reading. That this might be on the right track is confirmed by the well-established contrast between externally and internally headed relative clauses in Japanese pointed out by Shimoyama (1999). We will return to this issue in Section 5.2.

\subsection{The two analyses}

On the basis of the data just described, Cecchetto, Geraci \& Zucchi (2006) and Branchini \& Donati (2009) proposed two different analyses: In a nutshell, they are analyzed as correlatives by the former, but as internally headed relative clauses by the latter authors. We believe that if we set apart the restrictive/appositive contrast just mentioned above, this disagreement is more taxonomic than really factual. The main difference concerns the relation between the two clauses involved in the construction: According to the correlative analysis, the PE-clause is base-generated as adjoined to the main clause; according to the internally headed relative clause analysis, the PE-clause is generated in the position of the gap inside the main clause and later extraposed to the left edge of the main clause. We will not have much to add concerning this question here. Suffice it to say that embedded clauses are always extraposed in LIS, either to the left or to the right (see Cecchetto, Geraci \& Zucchi (2006) for a principled explanation and Geraci, Cecchetto $\&$ Zucchi (2008) for a possible exception involving control structures): This means that the sentence initial position of the PE-clause does not seem to be a feature especially related to relativization. Given this observation, we will set this issue aside in what follows. As for other differences between the analyses, we will show that these diverging views get reconciled once we adopt a new approach to relativization in general. This is what we will do in the next section. 


\section{A theory of relativization}

Relatives are clauses with a nominal distribution, involving A'-movement and acting as strong islands for extraction. In the framework of a theory of labeling we will briefly introduce in the next section, Cecchetto \& Donati (2010, 2015) and Donati \& Cecchetto (2011) provide a principled account for these three properties of relativization, connecting them as three effects of the same phenomenon: Head movement and its relabeling properties.

\subsection{Movement and label}

The starting point is the notion of Label as in (15) and the Probing Algorithm in (16) as defined by Cecchetto \& Donati (2010) (but see Adger (2003), Boeckx (2008), Chomsky (2008), and Pesetsky \& Torrego (2006) for similar proposals).

(15) Labels: When two objects a and $b$ are merged, a subset of the features of either $\mathrm{a}$ or $\mathrm{b}$ become the label of the syntactic object $\{a, b\}$. A label:

(i) can trigger further computation;

(ii) is visible from outside the syntactic object $\{a, b\}$.

(16) Probing Algorithm: The label of a syntactic object $\{a, b\}$ is/are the feature(s) which act(s) as a Probe of the merging operation creating $\{a, b\}$.

What (16) says is that the label of the category resulting from merge is always the feature asymmetrically triggering the merging operation. The core cases traditionally described by X-bar theory can be derived from (15) and (16) if the following assumptions are made: (i) words can be defined as the output of the morphology module and the input to the syntax module (cf. Cecchetto \& Donati (2015, chapter 1) for a defense of this traditional definition from recent criticism based anti-lexicalist frameworks), and (ii) a word has a property that forces it to merge with other categories to form phrases and clauses. This property of words (a sort of "special glue", called 'edge feature' by Chomsky (2008)) is the driving force, or technically the Probe, of the operation that merges a word with another category. So, assuming the Probing Algorithm in (16), any time a word is merged, it qualifies as a Probe. This means that a word, being a Probe by definition, can provide the label ("project" in traditional terms). For example, each time a head (= a word) is externally merged with its complement, the head is bound to project. 
This way, the system based on (16) captures the two empirical generalizations that any version of phrase structure theory must account for: Namely, that the target of movement (a Probe) typically projects and that a lexical item (a word) projects when it is merged with a phrase. Crucially for what follows, even when a lexical item is internally merged, i.e. moved, it can project.

\subsection{Head movement creates labeling ambiguities: free relatives}

We will call 'head movement' the movement of a word (as opposed to the movement of a phrase). It should be clear that this minimally simple notion of head movement is distinct from the technical notion of head movement in the Government and Binding framework. Given the Probing Algorithm, head movement is special, since it can "relabel" the landing site of movement.
a. I wonder what you read.
b. I read what you read.

In (17), a wh-word, 'what', is internally merged to a Probing C. The Probing Algorithm (16) correctly predicts that there should be a labeling ambiguity here. If the word provides the label, the structure ends up being a DP, i.e. a free relative; if the probing $\mathrm{C}$ provides the label, the structure is a (interrogative) clause: As a result, the structure is systematically ambiguous, as shown by its compatibility both with verbs selecting for nominal complements (e.g. 'read' in 17b) and with verbs selecting for clauses, as in (17a).

No ambiguity arises when a phrase is wh-moved: 'what book' in (18) does not qualify as a Probe, and only the target $\mathrm{C}$ is bound to project. (18) can only be an (indirect) interrogative clause.

(18) What book you read.

a. I wonder what book you read.

b. *I read what book you read.

Crucially, the phrasal/word status of the moving category is the only relevant difference between (17) and (18) (we refer to Donati \& Cecchetto (2011) for discussion of cases like "I read whatever book you suggest", which are shown to have properties that assimilate them to full relatives). wh-movement in (18) is probed in the same way (by a probing $\mathrm{C}$ searching for a $w h$-feature), and displays the same restrictions. For example, it can apply long distance (provided that it obeys familiar locality conditions): 
(19) a. I wonder/read what you told me that I should read what.

b. I wonder/*read what book you told me that I should read what book.

3.3. A relabeling raising analysis for externally headed relative clauses

As we argued in previous work (Donati \& Cecchetto 2011), externally headed full relatives can also be fruitfully analyzed as involving movement of a head that relabels the structure, as in (20).

(20) I like the $\left[_{N}\right.$ book $\left[_{C}\right.$ which book $\left[{ }_{T}\right.$ John read $\left[{ }_{D}\right.$ hich book $\left.\left.\left.]\right]\right]\right]$

In (20), first the phrase 'which book' wh-moves to the edge; then, and this is the crucial step, 'book' moves further, yielding target relabeling: What moves is a $\mathrm{N}$ and the structure gets a $\mathrm{N}$ label, in accordance with the Probing Algorithm (16). This label matches the selection requirements of the externally merged $\mathrm{D}$. This analysis inherits all the pros of the traditional raising analysis (cf. Vergnaud 1974; Kayne 1994; Bianchi 1999; Bhatt 2002; among others) as the external head noun and the gap are transformationally related. Furthermore, it has the merit of explaining for free the fundamental properties of relative clauses, namely that they are clauses with a nominal distribution.

Of course, the analysis proposed by Donati \& Cecchetto (2011) seems to face a problem when the external head of the relative clause is a phrase, as in $(21)$.

\section{(21) I like the book about Obama that John read.}

They assume that the material that modifies the head noun ('about Obama' in (21)) can (and must be) late-merged, after the head noun has moved and has "relabeled" the structure. This assumption makes so-called complements of nouns and adjuncts to the nouns more similar than it is usually thought. However, there is independent evidence that nouns do not take complements the same way verbs do. Since this is not the focus of this paper (remember, LIS PE-clauses are still waiting for an account!), we will simply refer to Donati \& Cecchetto (2011) for a detailed review of a number of arguments in favor of this conclusion (cf. Cecchetto \& Donati (2015, chapter 4) for psycholinguistic evidence going in the same direction). 


\section{LIS as the missing link: internally headed with $D$ relabeling}

Rather than pursuing further the analysis of English-like externally headed relative clauses, we will now return to PE-clauses and see whether the approach to relativization in terms of relabeling can do any good to our understanding of the LIS construction.

Remember that PE-clauses are head internal, namely the $\mathrm{N}$ that is modified by the PE-clause is in its canonical position inside the PE-clause (Section 2.1).

An advantage of (any version of) the raising analysis is that it accounts straightforwardly for the existence of internally headed relative clauses, which simply realize overtly what the raising analysis takes to be the underlying structure of externally headed relative clauses. This feature of the raising analysis should not be underestimated. While the raising analysis can explain the existence of two related relativization strategies by simply assuming that the "head" can raise at different point (before or after Spell-Out), alternative approaches to relative clauses have a harder time to explain why relativization can be realized through two different structures.

However, the peculiar version of the raising analysis we have been discussing so far can lead us much further in the comprehension of the LIS construction. Recall that we also know that LIS PE-clauses are closed by a determiner-like element sitting in the COMP area (Section 2.3), which moves there from the position of the head noun inside the PE-clause (Section 2.4).

It seems very reasonable to interpret this movement as yet another instance of a relabeling movement: $\mathrm{D}$ moves to $\mathrm{C}$ and relabels the structure, turning the clause into a (complex) DP: A relative clause. Having said this, LIS provides a sort of a missing link in the typology of relativization strategies we have discussed so far: It displays a construction which is something like a free relative, but with a full head in situ. The movement of PE is indeed very similar to the one involved in free relatives, being the movement of a $\mathrm{D}$ head; but the PE-clause is also a full relative, since it contains a full $\mathrm{N}$ head. In PE-clauses, the relabeling movement is a D-movement stranding the head noun in its base position. This also explains why PE can never pied-pipe the 'head': Recall that only lexical items, i.e. words, can project given the labeling algorithm (16).

The analysis for (22) is schematized in (23).

$\frac{b r}{\text { BOY }_{i} \text { CALL PE }_{\mathrm{i}}} \quad$ LEAVE DONE




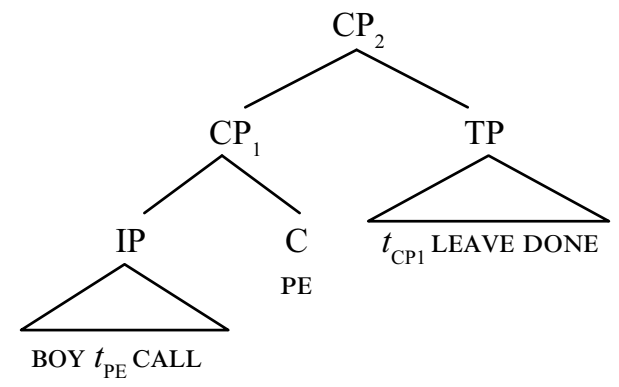

As we mentioned at the end of Section 2.7, we assume that PE-clauses are extraposed from their embedded position, as is generally the rule for embedded clauses in LIS: This is signaled in (22) by putting the trace $t_{\mathrm{CP} 1}$ in the position of the gap in the main clause.

\subsection{The trigger of PE-movement}

LIS displays both subject relatives and object relatives. In the object relative (24), the head noun BOY is the direct object of the PE-clause.

(24) GIANNI BOY B HIT $_{j} \frac{\text { br }}{\operatorname{PE}_{j} \text { MARIA }_{\mathrm{i}} \text { KISS }_{\mathrm{j}}}$
'Maria kissed a boy who Gianni hit.'

Given that we know that PE has moved to COMP in (24), this means that the subject GIANNI does not act as an intervener for the Probe-Goal relation established between the base position of PE (the internal head) and the $\mathrm{C}$ area. Still, the subject c-commands the base position of PE. So, assuming Relativized Minimality (Rizzi 1990), we are forced to conclude that PE bears some feature, some morphological property, that is probed by $\mathrm{C}$ and that a normal subject is not endowed with. What kind of feature can this be? Notice that PE is not a $w h$-element (it never shows up in questions), thus the familiar $w h$-feature cannot be invoked here. There is however another obvious candidate for the relevant feature, and this is the non-manual marking 'br' that obligatory accompanies relativization. Remember that 'br' is always realized at least in concomitance with $\mathrm{PE}$, optionally spreading over the entire clause: We will assume that this marking is indeed part of the feature endowment of PE itself, and qualifies it as a Goal for the probing COMP.

Something more should be said, beyond the purely descriptive 'raised eyebrows' label we gave to this feature. It is very possible that TOPIC is 
the relevant formal feature, since, as Branchini $(2007,2014)$ discusses in detail, 'br' is systematically present in (other) topicalization constructions. Incidentally, notice that even the morphological feature that is shared by wh-elements and is responsible for wh-movement in LIS, which is realized as 'wh' in English, must correspond in LIS to a non-manual marking. The $w h$-signs WHO, WHICH, WHEN, WHERE, WHY, WHAT do not share any manual feature in LIS: Neither location, nor hand configuration, nor movement. The only feature they have in common is the non-manual marking they co-occur with in questions, namely furrowed eyebrows.

An interesting question is how the dependency between COMP and PE is established in cases like (9a) above, in which PE does not overtly move. Covert movement of $\mathrm{PE}$ is an option. Some indirect evidence for this hypothesis comes from a fact observed by Branchini (2007, 2014), namely that spreading of the 'br' becomes obligatory when PE remains in situ. We argue in Section 4.4 below that when PE provides the label, the feature 'br' extends from PE to the entire structure it labels. That is, spreading of the 'br' feature indicates that in (9a) PE labels the structure over which 'br' spreads. We can even hypothesize that PE is allowed not to move overtly in (9a) because spreading of 'br' explicitly indicates that it will move and label the structure. Hence, non-manual marking may render overt movement non-obligatory.

\subsection{Predicting a structural ambiguity}

If the probe of the movement of $\mathrm{PE}$ is COMP, the relabeling analysis of PE-movement we have just presented makes one interesting prediction: The structure should be ambiguous, instantiating a case of probing "conflict" analogous to the one we discussed in Section 3.2 in relation to free relatives. PE can provide the label because it is a lexical item (word/sign) and a word is an intrinsic Probe. This is why PE-clauses can be relative clauses; but the Probe of the movement of PE is C, which can also provide the label, under the Probing Algorithm in (16). PE-clauses are thus predicted to have in this case a different, clausal, distribution. This prediction appears to be confirmed, as illustrated by (25) and (26).

(25) PIERO CONTRACT SIGN DONE $\frac{\mathrm{br}}{\text { PE GIANNI FORGET }}$
'Gianni forgot that Piero signed the contract.' 
(26)

br

PIERO CONTRACT $_{i}$ SIGN DONE PE $_{i}$ GIANNI FORGET

'Gianni forgot the contract that Piero signed.'

(25) is minimally different from (26). In fact, if one looks only at the order of manual signs, the two sentences might appear to be the same sentence. However, there are differences: In (25), unlike what happens in (26), PE is not articulated in the same position in space as CONTRACT (in the glosses this is indicated by the absence of co-indexing). Our informants argue that PE does not refer to the noun CONTRACT in (25) but to the entire clause PIERO CONTRACT SIGN DONE ('Piero signed the contract').

The second difference is that 'br' naturally spreads over the entire clause in (26), while it is restricted to PE in (25). Finally there is a clear interpretative difference: The PE-clause is interpreted as a relative clause in (26) but as a complement clause in (25), as indicated by the different translations. The existence of embedded declarative clauses doubled by PE was already noticed by Geraci, Cecchetto \& Zucchi (2008: 57), who analyze PE as a resumptive pronoun in these structures but comment: "Interestingly, the form of the resumptive pronoun is identical to the relative element used in the (cor)relative construction in LIS discussed in Cecchetto, Geraci \& Zucchi (2006) and Branchini \& Donati (2009).”

The relabeling approach to relativization can explain why the same element is used in embedded relatives and in embedded declaratives, and allows us to unveil a parallelism with the free relatives case. More explicitly, what we propose is that the derivation of (25) and (26) might be the same, but for the fact that, after PE has moved to the COMP area, the Probe (COMP) provides the label in (25), while in (26) the Goal (namely, PE itself) does. So, the source position of PE would be the same in (25) and (26): Namely $\mathrm{PE}$, being a determiner-like element, is adjacent to the noun CONTRACT in the input structure of both sentences. Under this analysis, PE is a category that can act as a determiner (this happens in its base position and, limitedly to (26), also in its derived position) but can also act as a complementizer (this happens in its derived position in (25)). Needless to say, the pattern in which the same word/sign has a double life as a determiner and as a complementizer is not at all uncommon cross-linguistically, one obvious example being English 'that' (cf. "that (one)" / "I believe that ...").

Although the parallelism with free relatives is remarkable, there is an important difference. In the free relative case, there can either be a relative clause interpretation (when the $w h$-word projects) or an embedded question interpretation (when COMP does). However, PE is not a wh-element, hence 
the ambiguity is a different one: The PE-clause can either be a relative clause (when PE projects, as in (26)) or a declarative complement clause (when COMP does, as in (25)).

Before further commenting on the non-relative use of $\mathrm{PE}$, let us introduce a caveat: Due to the importance of minimal pairs like the one in (25) and (26) for our theory of relativization, when writing this paper we contacted again the informant of Geraci, Cecchetto \& Zucchi (2008) who originally produced sentences like (25). While he fully confirmed his original judgments, he pointed out to us that the non-relative use of PE is possible only with same types of matrix verbs. For example, this use is fully felicitous with matrix verbs like FORGET, as in (25), while he found the structures degraded if the matrix verb is THINK. Although this aspect deserves further analysis, we suspect that the non-relative use of PE requires that the verb selecting the embedded clause doubled by PE be factive. Therefore, a better translation of (25) might be "Gianni forgot the fact that Piero signed the contract".

\subsection{PE in the main clause}

As soon as we recognize (25) and (26) as a minimal pair, other interesting differences emerge. One of them is that PE can also be stranded in the main clause in sentences like (27), where the subordinate clause is dislocated to the left, as usual. The stranding of PE is never attested when the clause has a relative interpretation (cf. the ungrammaticality of (28)).

PIERO CONTRACT SIGN DONE GIANNI $\underline{\mathrm{br}}$ PE FORGET
'Gianni forgot that Piero signed the contract.'

\footnotetext{
*PIERO CONTRACT SIGN DONE GIANNI PE FORGET 'Gianni forgot the contract that Piero signed.'
}

This sharp contrast can be explained given our approach to labeling. In relative clauses, PE is the label of the clause, and as such cannot be stranded for the same reason one cannot strand COMP when dislocating the clause it heads, as is illustrated by the contrast between (29a) and (29b).

(29) a. Whether Paul is a liar, John wonders.

b. *Paul is a liar, John wonders whether. 
In cases like (27), the label of the clause is $C$, the Probe of the movement of $\mathrm{PE}$ to the COMP area. If PE is adjoined to the COMP area, its stranding is possible. PE then plausibly assumes a resumptive role.

\subsection{The spreading of the non-manual marking}

Let us now turn to another difference in our minimal pair (25) vs. (26) concerning the extension of the non-manual marking associated with PE, 'br'. As we mentioned, while 'br' naturally spreads over the entire clause when the PE-clause has a relative interpretation, it is restricted to PE in sentences like (25) or (27), namely when the PE-clause is an embedded clausal complement.

Interestingly, the kind of spreading we observe in relativization is quite different from the one displayed in wh-questions. Consider (30) and (31).

br

PAOLO MARIA IDEA SUGGEST PE $_{\mathrm{i}}$ IMPORTANT

'The idea that Paolo suggested to Maria is important.'

wh

PAOLO STEAL BOOK WHICH $_{\mathrm{i}}$ 'Which book did Paolo steal?'

In questions, as described thoroughly in Cecchetto, Geraci \& Zucchi (2009), the extension of the spreading is directly related to the dependency established through $w h$-movement: If the $w h$-element is moved from an object position, the non-manual marking will spread from the object on, crucially excluding the preverbal subject (31). In relatives, on the other hand, spreading, when it occurs, is always on the entire clause, no matter where the PE starts from: In (30) it raises from the object position and the spreading of 'br' does not match its path, since it spreads over the preverbal subject as well.

There are two different ways to go to explain the pattern of non-manual marking associated to PE-clauses. One way, suggested by a reviewer, involves abandoning the idea that the ambiguity in (25)-(26) is related to the labeling configuration emerging from movement of PE. According to the reviewer's suggestion, while the relative structure in (26) results from movement of $\mathrm{PE}$, as we have been arguing in this paper, PE in (25) might be a nominalizer particle directly merged in the COMP position, where it can label the structure by virtue of being a word/sign. Since in (25) there would be no chain 
of PE to begin with, it is expected that non-manual marking is restricted to PE. While we acknowledge that this is a possible analysis for (25), we would like to stress that the pattern of non-manual marking can also be naturally explained by the proposal that the ambiguity in (25)-(26) is contingent on what labels the structure after movement of PE. In fact, non-manual marking spreading in PE-clauses may be a direct consequence of the labeling movement that we claim takes place here: 'br' is a feature of the sign PE. It goes with PE itself. When PE labels (and nominalizes) the clause, projecting to CP, the feature marks the clause as well. When no such labeling happens (as in the minimally different complement clause we are discussing), no projection of the feature is possible and the non-manual marking has to remain local on PE. As for $w h$-questions, what might appear as a similar fact, the extension of the non-manual marking, is indeed a rather different phenomenon, not due to projection (the $w h$-sign does not label the structure in (31)).

\section{Some cross-linguistic explorations}

The analysis we have discussed in the previous section seems to be able to put together all the properties of LIS PE-clauses we described in Section 2 , while at the same time it makes interesting and accurate predictions on a minimally different construction. We think that this analysis has also the merit of opening interesting questions along the cross-linguistic and typological dimension. Let us now conclude by briefly discussing some promising directions this analysis appears to open.

\subsection{When a D can strand its complement (and when it cannot)}

A first advantage of the approach we took here is that it allows us to shed light on some interesting restrictions on movement in general. In particular, we might want to ask the following: Why isn't the structure underlying PE-clauses attested elsewhere, like, say, in English? More precisely, we might want to know why something like (32) is not possible in English.

*I will eat what you will eat wat cookie.

(32) would be the equivalent of LIS PE-clauses, displaying the movement of a D-head ('what') stranding its nominal complement (the only relevant difference being that the category that moves is a $w h$-word in the putative English 
example). (32) is sharply ungrammatical. Notice that the ban on stranding the restriction of $\mathrm{D}$ is not limited to relative environments, but it is rather a general fact in English. (33) contains an indirect question moving 'what' and stranding 'cookie', and the result is again completely unacceptable.

* I wonder what you will eat what cookie.

That the ability of stranding the restriction of $\mathrm{D}$ is language-specific and not construction-specific is confirmed by data like (34), which show that in LIS also $w h$-elements can be moved alone stranding their complement. ${ }^{2}$

$\begin{array}{ll} & \frac{w h}{} \\ & \\ \text { 'Which bread did Paola eat?' }\end{array}$

It has been observed that the possibility of extracting a wh-element out of a nominal expression is directly related to agreement: We discuss this hypothesis in details in Cecchetto \& Donati (2010). Just to illustrate this correlation, let us focus on Italian, which displays a strong contrast in questions. Pied-piping is obligatory when the $w h$-element agrees with its correlate (35), while it is optional (and even dispreferred according to some speakers) when it does not (36).
a. Quant-i libr-i hai letto? how-many-PL book-PL have.2SG read
b. *Quant-i hai letto libr-i? 'How many books have you read?' how-many-PL have.2SG read book-PL
a. Quant-o hai detto che sono alt-i? how-SG have.2SG said that (they) be.3SG tall-PL
b. ?Quant-o alt-i hai detto che sono? how-SG tall-PL have.2SG said that (they) be.3SG 'How tall did you say that they are?'

In Cecchetto \& Donati (2010), we relate this correlation to labeling once again: In a nutshell, the idea is that agreement, being a form of probing, forces D to label the structure. As such, the label D is always closer to the probing $\mathrm{C}$ than the head $\mathrm{D}$, hence forcing pied-piping. When no agreement 
holds, on the other hand, D does not have to label the structure and the head $\mathrm{D}$ can be the closest goal for the probing $\mathrm{C}$.

Going back to LIS, if we are on the right track, we might have here a good reason to conclude that what is general described as 'agreement in space' for sign languages in not the same kind of relation that we observe in gender or number agreement in spoken languages: As a matter of fact, while that kind of agreement blocks D movement, agreement in space does not (on a slightly different approach to $w h$-splitting in LIS, see Geraci \& Cecchetto (2013)).

\subsection{Internally head relative clauses: back to typology}

Japanese displays two types of relative clauses: An externally headed construction (37a) similar to, say, English; and an internally headed construction, which is strongly reminiscent of LIS (37b).

a. Yoko-wa [[Taro-ga sara-no ue-ni oita]keeki]-o tabeta. Yoko-TOP [[Taro-NOM plate-GEN on-LOC put] cake]-ACC ate 'Yoko ate a piece of cake which Taro put on a plate.'

b. Yoko-wa [[Taro-ga sara-no ue-ni keeki-o oita]-no]-o tabeta. Yoko-TOP [[Taro-NOM plate-GEN on-LOC cake-ACC put]-NMLZ]-ACC ate 'Yoko ate a piece of cake which Taro put on a plate.'

(Shimoyama 1999: 147)

Interestingly, when the relative clause head keeki does not move (namely in the internally headed relative in (37b)), the nominalizer particle no surfaces in the right periphery of the relative clause. This particle is not present when keeki moves. In our relabeling approach, this can be interpreted as an indication that in (37a) keeki moves to the structural position that is occupied by the nominalizer particle in (37b). The particle is not needed in (37a), since the movement of keeki can relabel the structure by turning it into a nominal constituent.

But the similarity of LIS PE-clauses with Japanese internally headed relatives goes even further, and involves interpretation. Remember that relatives in LIS display an unexpected, 'non-restrictive' interpretation, when containing a universal quantifier modifying the head (Section 2.6). The very same fact is discussed in Shimoyama (1999), and is illustrated in (38). 
(38)

Taro-wa [[Hanako-ga dono sinbun-mo katte kita]-no]-o

Taro-TOP [[Hanako-NOM every newspaper buy came]-NMLZ]-ACC tana-ni narabeta.

shelf-on placed

'Hanako bought (and brought) every newspaper and Taro shelved them.'

(Shimoyama 1999: 166)

There is a crucial difference between a standard raising analysis and our relabeling analysis: We are not claiming that internally headed relatives, like PE-clauses or the Japanese clauses illustrated by (38), are the covert movement counterpart of externally headed relatives. Our idea is that what moves in internally headed relatives is a determiner, while what moves in externally headed relative clauses is a noun (with the determiner externally merged to the clause itself; see Section 3.3). This means that we are not predicting that the two constructions have exactly the same interpretation. And this is a good thing in light of the fact illustrated in (38) and in Section 2.6 above for LIS. As for the exact explanation to be given to the non-restrictive reading displayed in Japanese and LIS, we conjecture that what Shimoyama proposes for Japanese can be extended to LIS: Namely that no (PE in LIS), in addition to being the nominalizer of the clause, also and crucially introduces an e-type pronoun.

A reviewer asks what explains the fact that while Japanese has both internally headed and externally headed relatives, LIS displays only the former. Answering this type of cross-linguistic questions is not easy and we acknowledge that we do not have a comprehensive answer. However, we can point out a factor that is likely to play a role. In LIS, PE is generated next to the head noun, while in Japanese there is no evidence that a determiner-like element must be generated next to the relative clause head. A consequence is the following. If in LIS the phrase composed of noun + PE moved, a problem would arise. A phrase (as opposed to a word/sign) does not have a relabeling power, given the theory of labeling sketched in Section 3.1 above. Therefore the structure resulting from movement of noun + PE could not be a nominal constituent. On the other hand, movement of $\mathrm{N}$ without PE is likely to involve a minimality violation, as in cases in which a noun is extracted from a DP and the determiner is stranded. In Japanese, however, these complications do not arise, since the head noun can move alone and relabel the structure, as in (37a) above. ${ }^{3}$

Finally, notice that the correlation between head internal relatives and nominalizer-/ determiner-like particles is not restricted to Japanese, but is very widely attested cross-linguistically (Keenan 1985; Comrie 1981). Given 
this clear correlation, it would be interesting to verify whether the analysis in terms of relabeling movement we have proposed for LIS could be extended to other internally headed relatives in other languages.

\subsection{Sign languages: cross-linguistic perspectives}

Let us close by briefly addressing the issue from the perspective of sign languages typology. Branchini et al. (2007) have shown that sign languages adopt various relativization strategies, including externally headed relative clauses - be they introduced by a relative pronoun (as in German Sign Language (DGS), American Sign Language (ASL)) or not (as in Brazilian Sign Language (LSB) - and internally headed relative clauses (attested, for instance, in ASL, Israeli Sign Language (ISL), Hong Kong Sign Language (HKSL), Turkish Sign Language (TID), and, of course, LIS). This complex and varied picture confirms once again the cross-modal validity of the typological categories, and seems to suggest that the same range and amount of variation observable for spoken languages is also displayed across sign languages.

\section{Notes}

1. There is mismatch between the acronym LIS and the full name of the language in Italian (Lingua dei Segni Italiana). For the history of the language and its name cf. Volterra (2011).

2. Notice that our approach predicts that structures such as (33), displaying the head movement of a wh-element, should also be ambiguous in LIS, just as free relatives in English are; see Branchini (2012) for an interesting exploration of this prediction.

3. Remember that Donati \& Cecchetto (2011) analyze sentences like (21), repeated as (i), as cases of late merge of the modifier to the noun after the noun has moved alone and relabeled the structure.

(i) I like the book about Obama that John read.

The arguments they offer in favour of this late merge analysis do not extend to the case of a DP. So, under their analysis, it is not expected that a D can move alone, relabel the structure, and be supplemented by a noun only at this late stage. An alternative for building externally headed relative clauses in languages displaying determiners is of course the one instantiated in English and other European languages and discussed in Section 3.3. Here relativization happens in two steps thanks to the $w h$-feature: First the entire DP phrase is $w h$-moved, and then the noun alone is extracted and relabels the clause, nominalizing it. 


\section{References}

Adger, David. 2003. Core syntax. Oxford: Oxford University Press.

Bhatt, Rajesh. 2002. The raising analysis of relative clauses: Evidence from adjectival modification. Natural Language Semantics 10. 43-90.

Bianchi, Valentina. 1999. Consequences of antisymmetry: Headed relative clauses. Berlin: Mouton de Gruyter.

Boeckx, Cedric. 2008. Bare syntax. Oxford: Oxford University Press.

Branchini, Chiara. 2007. On relativization in Italian Sign Language. PhD dissertation, Università di Urbino.

Branchini, Chiara. 2012. A note on LIS free relatives. Poster presented at Formal and Experimental Advances in Sign Language Theory (FEAST 2012), University of Warsaw.

Branchini, Chiara. 2014. On relativization and clefting: An analysis of Italian Sign Language (LIS). Berlin: De Gruyter Mouton.

Branchini, Chiara \& Carlo Geraci. 2011. L'ordine dei costituenti in LIS: risultati preliminari. In Anna Cardinaletti, Carlo Cecchetto \& Caterina Donati (eds.), Grammatica, lessico e dimensioni di variazione nella LIS, 113-126. Milano: Franco Angeli.

Branchini, Chiara \& Caterina Donati. 2009. Relatively different. Italian Sign Language relative clauses in a typological perspective. In Aniko Liptàk (ed.), Correlatives cross-linguistically, 157-191. Amsterdam: John Benjamins.

Branchini, Chiara, Caterina Donati, Roland Pfau \& Markus Steinbach. 2007. A typological perspective on relative clauses in sign languages. Talk presented at $7^{\text {th }}$ Conference of the Association of Linguistic Typology (ALT), Paris, September 2007.

Cecchetto Carlo, Carlo Geraci \& Sandro Zucchi. 2006. Strategies of relativization in Italian Sign Language. Natural Language and Linguistic Theory 24. 945-975.

Cecchetto Carlo, Carlo Geraci \& Sandro Zucchi. 2009. Another way to mark syntactic dependencies. The case for right-peripheral specifiers in sign languages. Language 85. 1-43.

Cecchetto, Carlo \& Caterina Donati. 2010. On labeling: Principle C and head movement. Syntax 13. 241-278.

Cecchetto, Carlo \& Caterina Donati. 2015. (Re)labeling. Cambridge: MA: MIT Press.

Chomsky, Noam. 2008. On phases. In Robert Freidin, Carlos P. Otero \& Maria Luisa Zubizarreta (eds.), Foundational issues in linguistic theory, 133-166. Cambridge, MA: MIT Press. 
Comrie, Bernard. 1981. Language universals and linguistic typology: Syntax and morphology. Chicago: University of Chicago Press.

Donati, Caterina \& Carlo Cecchetto. 2011. Relabeling heads: A unified account for relativization structures. Linguistic Inquiry 42. 519-560.

Geraci, Carlo \& Carlo Cecchetto. 2013. Neglected cases of rightward movement. When wh-phrases and negative quantifiers go to the right. In Heike Walker, Gert Webelhuth \& Manfred Sailer (eds.), Rightward movement in a comparative perspective, 211-241. Amsterdam: John Benjamins.

Geraci, Carlo, Carlo Cecchetto \& Sandro Zucchi. 2008. Sentential complementation in Italian Sign Language. In Michael Grosvald \& Dionne Soares (eds.), Proceedings of the $38^{\text {th }}$ Western Conference on Linguistics, 46-58. Davis, CA: California State University.

Kayne, Richard. 1994. The antisymmetry of syntax. Cambridge, MA: MIT Press.

Keenan, Edward. 1985. Relative clauses. In Timothy Shopen (ed.), Language typology and syntactic description II: Complex constructions, 141-170. Cambridge: Cambridge University Press.

Pesetsky, David \& Esther Torrego. 2006. Probes, goals and syntactic categories. In Yukio Otsu (ed.), Proceedings of the $7^{\text {th }}$ Tokyo Conference on Psycholinguistics, 25-60. Tokyo: Hituzi Syobo.

Rizzi, Luigi. 1990. Relativized minimality. Cambridge, MA: MIT Press.

Shimoyama, Junko. 1999. Internally headed relative clauses in Japanese and E-type anaphora. Journal of East Asian Linguistics 8. 147-182.

Vergnaud, Jean Roger. 1974. French relative clauses. PhD dissertation, Massachusetts Institute of Technology.

Volterra, Virginia. 2011. La ricerca sulla lingua dei segni in Italia: passato, presente e prospettive future. In Anna Cardinaletti, Carlo Cecchetto \& Caterina Donati (eds.), Grammatica, lessico e dimensioni di variazione nella LIS, 27-44. Milano: Franco Angeli.

Zucchi, Sandro, Carlo Neidle, Carlo Geraci, Quinn Duffy \& Carlo Cecchetto. 2010. Functional markers in sign languages. In Diane Brentari (ed.), Sign languages (Cambridge Language Surveys), 437-492. Cambridge: Cambridge University Press. 